\title{
Bi-objective optimization of a grid-connected decentralized energy system
}

\author{
Onur Altıntaş | Busra Okten | Özlem Karsu@ | Ayse Selin Kocaman
}

Department of Industrial Engineering, Bilkent University, 06800 Ankara, Turkey

Correspondence

Özlem Karsu, Department of Industrial

Engineering, Bilkent University, 06800

Ankara, Turkey.

Email: ozlemkarsu@bilkent.edu.tr

\begin{abstract}
Summary
Motivated by the increasing transition from fossil fuel-based centralized systems to renewable energy-based decentralized systems, we consider a bi-objective investment planning problem of a grid-connected decentralized hybrid renewable energy system. In this system, solar and wind are the main electricity generation resources. A national grid is assumed to be a carbon-intense alternative to the renewables and is used as a backup source to ensure reliability. We consider both total cost and carbon emissions caused by electricity purchased from the grid. We first discuss a novel simulation-optimization algorithm and then adapt multi-objective metaheuristic algorithms. We integrate a simulation module to these algorithms to handle the stochastic nature of this bi-objective problem. We perform extensive comparative analysis for the solution approaches and report their performances in terms of solution time and quality based on well-known measures from the literature.
\end{abstract}

\section{KEYWORDS}

bi-objective programming, $\mathrm{CO}$ emission, grid-connected decentralized systems, metaheuristic algorithms, renewable energy, simulation-optimization, 2-stage stochastic mixed-integer programming

\section{1 | INTRODUCTION}

Global warming has become one of the biggest concerns of the 21st century and will be a major issue in the following centuries. It is known that the main reason for global warming is society's increase in greenhouse gas emissions. Among all greenhouse gases, carbon dioxide $\left(\mathrm{CO}_{2}\right)$ is the biggest driver of global warming.

The reason for current high-carbon emission rates is society's dependency on electricity generation from fossil fuel-based centralized energy systems. In such systems, electricity is produced in large-scale (mostly thermal power) plants and distributed to the end user. Greenhouse gas emissions can be reduced significantly by shifting from centralized systems to decentralized ones that are based on renewable sources. In this regard, most countries promote decentralized systems that rely on renewable resources to decrease carbon emission levels and dependence on finite-fossil fuel reserves. ${ }^{1}$
One of the main drawbacks related to renewable energy systems is that renewable sources are only partially predictable and have limited controllability (ie, they are intermittent). To ease this difficulty, most decentralized energy systems include more than one type of energy resource, preferably with complementary availability patterns. ${ }^{2}$ Such systems are called hybrid energy systems. In the literature, many hybrid systems include renewable sources such as solar, wind or hydroelectricity, and storage technologies. ${ }^{1-3}$ Another drawback of renewable energy systems is that they heavily depend on the spatial location. Hence, decentralized systems can usually only be located in areas where renewable sources are available.

Decentralized systems could be designed as stand-alone (SA) or grid-connected (GC) systems. Stand-alone systems are generally located in remote places where grid networks cannot penetrate. These systems usually have drawbacks such as low capacity factors, renewable energy 
curtailment, or high storage costs. On the other hand, GC systems can be built on a large scale as they are connected to the main grid network. This connection enables the system both to purchase electricity from the grid network if there is not enough renewable energy to meet demand and to feed excess electricity to the grid (see Kaundinya et $\mathrm{al}^{3}$ for a review paper on decentralized systems).

While designing a GC decentralized system, policy makers and investors face the decision of choosing an optimal investment amount. One extreme solution is relying fully on fossil fuel-based energy, ie, electricity from the grid, which leads to fewer costs but higher emissions. Another solution is highly investing in renewables for emission minimization. Note that there are intermediate solutions between these 2 extreme solutions, in which demand satisfaction relies partly on renewables and partly on the grid.

In this study, we investigate the optimal sizing decision of a GC decentralized system, which consists of solar, wind, and storage units. In our setting, we assume that the decision maker has both cost and carbon emission concerns and that the investment size of the system will be determined depending on how carbon sensitive the decision maker is. Therefore, we take into account the multiple criteria that the decision maker will be considering and present the trade-off between cost and $\mathrm{CO}_{2}$ emission levels. To the best of our knowledge, our study is the first to provide a mathematical programming formulation along with novel simulation-optimization approach and metaheuristic algorithms for a multi-objective design of a GC decentralized energy system (GCDES) while incorporating the uncertainty of renewable resources. We perform an extensive comparative analysis of the proposed methods.

\section{2 | LITERATURE REVIEW}

With the increased awareness of global warming, the interest in decentralized energy systems (which mostly work with renewable energy sources) has increased in the literature. Jebaraj and Iniyan ${ }^{4}$ and Hiremath et $\mathrm{al}^{5}$ publish reviews on energy models in general and decentralized energy planning models, respectively. Kaundinya et $\mathrm{al}^{3}$ review SA and GC decentralized systems and explain their operational differences.

Most papers on hybrid renewable energy system (HRES) design and optimization are focused on SA HRESs. In this study, we review the literature on GC decentralized HRESs and categorize the problems as single objective ${ }^{6-9}$ and multi-objective. ${ }^{10-14}$ The review summary can be found in Table 1.

A methodology proposed by Chedid and Rahman ${ }^{10}$ finds the most favorable design for a decentralized system of which the electricity generation depends on solar and wind resources. Storage devices and diesel generators are also used in the system as backup sources. The authors analyze both SA (autonomous) and GC versions of the system. In this analysis, production cost of energy is minimized by using linear programming methods while taking environmental factors into consideration.

Wang and Singh $^{11}$ consider the multi-criteria design of a GC HRES. Their system includes photovoltaic panels, wind turbines, and battery units with a connection to the grid. In this setting, generated excess electricity cannot be fed back to the grid rather must be spilled, which restricts the system's component sizes. Three conflicting objectives are considered in this problem: minimizing cost, minimizing emission, and maximizing reliability (the ratio of meeting demand by renewables). The authors develop a multi-objective particle swarm optimization (MOPSO) algorithm to obtain a set of nondominated solutions.

Perera et $\mathrm{al}^{12}$ introduce a $\varepsilon$-multiobjective optimization technique to determine the optimal design of a GC hybrid solar/wind/storage system. They find the optimal component sizes with a minimum level of grid integration and energy cost. The results show that the levelized energy cost decreases when moving from SA mode to GC mode.

Sharafi and ElMekkawy propose a dynamic-MOPSO (DMOPSO) model for the design of a HRES, and Sharafi et al use a simulation-based DMOPSO model for optimal sizing of a GC HRES for residential buildings. ${ }^{13,15}$ In the GC system, 3 objective functions (minimizing total net present cost, minimizing $\mathrm{CO}_{2}$ emission, and maximizing renewable energy ratio) are used. The system uses solar, wind, and biomass as resources. In this setting, renewable energy is stored using a heat tank. Plug-in electric vehicles are also included in the system so that vehicles could be charged using renewable energy. The authors compare their results with the results of a multi-objective GA and a MOPSO algorithm. In this work, the uncertainty of renewable resources is not taken into account.

As mentioned above, because of the variability and intermittency of renewable resources, modeling systems with renewables is a challenging task. Therefore, in most optimal designs of decentralized energy systems, intermittent resources such as wind and solar are modeled using hourly average values for their availabilities. ${ }^{10-12}$ This method, however, results in losing information because peak values are not taken into account. ${ }^{16,17}$ Further, the variability and trend in renewables' availabilities cannot be captured by averages. Moreover, some studies only use 1 year of hourly data to capture seasonality and trends in resource availabilities and do not focus on uncertainty. $6,8,9$

A more realistic way of approaching renewable energy generation problem is to take uncertainty into consideration. Powell et al highlight the importance of modeling 


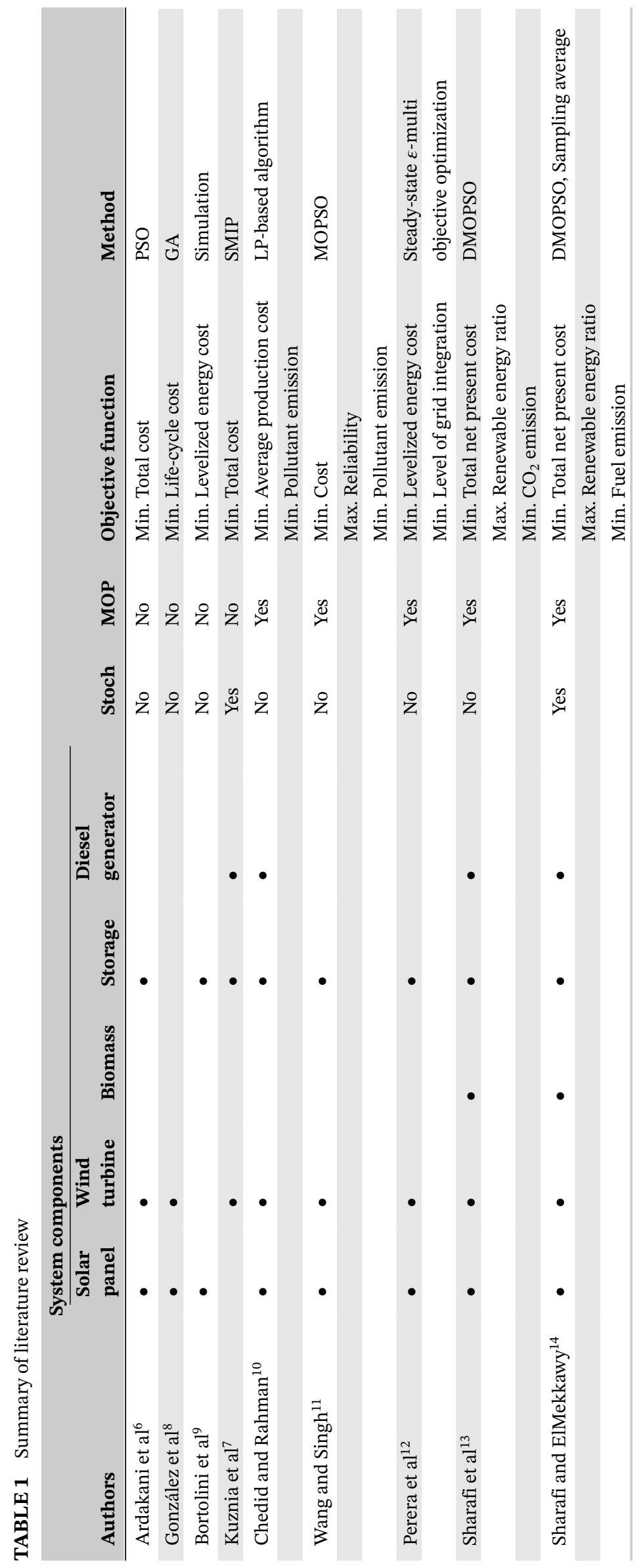


the uncertainty of renewable resources and discuss problems commonly encountered when doing so. ${ }^{16}$ In Kuznia et al, ${ }^{7}$ the optimal design problem is modeled using 2 -stage stochastic mixed-integer programming. One year of wind speed data is disintegrated into seasons. Then, the problem is solved using a variant of Benders' decomposition method. Sharafi and ElMekkawy ${ }^{14}$ include the stochasticity of renewable resources and variability in demand into the system that they propose in Sharafi et al. ${ }^{13}$ Pareto front is approximated using a simulation module, DMOPSO algorithm, and sampling-average method. The authors have 3 objectives: maximizing the renewable energy ratio, minimizing total net present cost, and minimizing fuel emission. Randomness is incorporated in the parameters using synthetic data-generation techniques. Stochastic and deterministic Pareto fronts are compared, and a sensitivity analysis is conducted.

To sum up, the 2 aspects that make these optimal design problems complex (their multi-objective and stochastic natures) should be considered to obtain more realistic results. Yet to the best of our knowledge, the work of Sharafi and ElMekkawy ${ }^{14}$ is the only study in the literature that considers a multi-objective design problem of a GCDES while handling the uncertainty related to renewable resources. We pursue a similar line of research but focus on a generic system, which includes all the challenges related to the system design. We provide a mathematical programming formulation along with a novel simulation-optimization approach. We also modify existing metaheuristic algorithms for the solution of this problem. Our methods handle the multi-criteria nature of the problem by considering the 2 conflicting criteria of cost and $\mathrm{CO}_{2}$ emissions as well as its multi-stage stochastic nature, via an integrated simulation-optimization framework.

\section{3 | PROBLEM DEFINITION}

In this study, we consider a framework in which a decision maker plans to invest in a decentralized system. We assume that the demand point (such as a village or a college campus) is already connected to the grid network, which is assumed to supply carbon-intense energy at a low price. The projected decentralized system is an HRES that consists of solar and wind power systems as well as a storage device to reduce the effect of the intermittency of renewables.

For wind power generation, 3 different wind turbine types are considered for investment in our problem. These turbines have different costs and rated powers, and investors can invest in one or multiple types. For solar power generation and storage systems, we do not explicitly specify the technology used; rather, we define the unit solar energy generation as a function of an efficiency parameter and the solar irradiation. Similarly, for the storage device, we use a generic efficiency parameter, which can be changed based on the technology used. We assume linear cost functions for the solar power generation and storage devices (ie, the cost of the unit size of these components is constant).

An HRES can be used either to satisfy local demand or to make a profit by selling green energy to the grid at elevated prices. In this study, we assume that the decision maker is carbon sensitive; hence, the priority of the decentralized system is to satisfy local demand using green energy rather than feeding energy to the grid to make a profit. If there is a surplus of renewable energy, it can be stored in the storage device and/or fed to the grid. We assume that the storage device can only store green energy and that this energy can only be used to satisfy the demand (ie, renewable energy cannot be sold to the grid through the storage device; therefore, we can prioritize renewable energy to be used for local demand). Fossil fuel-based electricity from the grid will be used as a backup source only when green energy is not adequate to meet the demand. A schematic description of the decentralized system can be found in Figure 1.

Governments impose different incentive policies, such as feed-in tariff programs, tax deductions, and investment and operating subsidies, to promote renewable energy investments and decrease $\mathrm{CO}_{2}$ emissions. ${ }^{18}$ We consider a setting where a feed-in tariff program is available to investors. This program is an incentive policy that aims to promote renewable energy investments by offering higher selling prices for each renewable energy type. Green

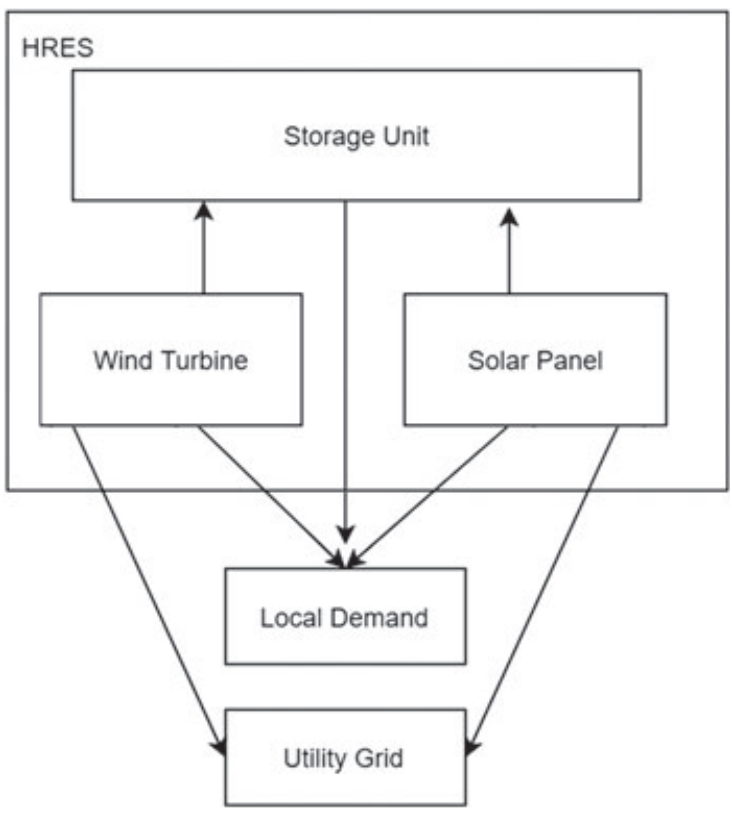

FIGURE 1 The grid-connected decentralized energy system 
energy can be sold to the grid for higher prices for a limited time..$^{18}$ It is expected that feed-in tariff programs will increase the ratio of clean energy fed to the grid in the long run. This situation will decrease the carbon emission rate of the electricity bought from the grid. However, in this study, we assume that this improvement is negligible.

The objective of this study is to determine the change in size of the described system with respect to the carbon emission value. To handle the 2 conflicting objectives considered (cost and $\mathrm{CO}_{2}$ emissions), we propose a solution framework in which we determine the optimal sizing of the components and their relations and present a set of solutions rather than a single solution. Our solution framework is generic, that is, independent of the system scale. Thus, it can be used for demand points of different sizes at different locations.

The decisions to be made in such systems are of 2 types: investment decisions and operational decisions. Investment decisions include sizing decisions for the components (solar panel area, number of wind turbines, storage size) and are made at the beginning of the time horizon. Operational decisions, on the other hand, are made in each time unit, such as deciding on the amount of energy to be stored, purchased, and/or sold. All these decisions are to be made considering both cost and emission criteria. Note that in addition to being bi-objective, the problem is stochastic because of the uncertainty of renewable resources. The decision support system we propose helps the decision maker to make investment decisions for such systems, taking into account the problem's multicriteria and stochastic natures.

\section{SOLUTION APPROACHES}

\section{1 | Bi-objective 2-stage stochastic mixed-integer programming approach}

In 2-stage stochastic programs, the decision-making process is divided into 2 stages, where there are 2 different types of decision variables: first and second stage. First-stage variables are decided on before the realization of random parameters. After uncertain events unfold (such as availability of renewable resources), operational decisions can be made. The general form of the 2-stage stochastic linear program is given below:

$$
\begin{aligned}
\text { Min } & c^{T} X+\mathbb{E}[Q(X, \xi(\theta))] \\
\text { s.t } & A X=b \\
& X \geqslant 0 \\
\text { where } & Q(X, \xi(\theta))=\operatorname{Min} q^{T} Y \\
\text { s.t } & t X+w Y=h \\
& Y \geqslant 0
\end{aligned}
$$

where $X$ and $Y$ are first- and second-stage variables, respectively. The second-stage problem depends on the data $(q, t, w, h)$, where some or all elements can be random. The expectation of $Q$ is taken with regard to the probability of $\xi$. Randomness in $\xi$ can be incorporated in 2 ways. The first way uses a continuous probability distribution. This approach keeps the problem size steady, but it may cause nonlinearities and computational difficulties. ${ }^{19}$ The second way is scenario based. In this approach, uncertainty is modeled as a union of random discrete events. There are a finite number of possible outcomes with certain probabilities, and the problem size increases enormously depending on the number of outcomes. Let $\Theta$ be the number of possible outcomes and $p_{\theta}$ be the corresponding occurrence probability of scenario $\theta$. Then, the 2-stage stochastic program with discrete random events becomes:

$$
\begin{array}{rlr}
\text { Min } & c^{T} X+\sum_{\theta=1}^{\Theta}\left[p_{\theta} q_{\theta} Y_{\theta}\right] \\
\text { s.t } & A X=b & \\
& t_{\theta} X+w_{\theta} Y_{\theta}=h_{\theta} \quad \theta=1 \ldots \Theta \\
& X \geqslant 0, Y_{\theta} \geqslant 0 & \theta=1 \ldots \Theta .
\end{array}
$$

We model our problem as a bi-objective 2-stage stochastic mixed-integer program. To be able to model the random availabilities of resources, we follow a scenario-based approach. Renewable energy generation depends on uncertain data such as wind speed and solar irradiation, and the sizing decision must be made before such uncertainties are realized. Once the component sizes of the decentralized system are determined, the amount of renewable energy generation can be calculated and operational decisions (storing, outsourcing, and meeting local demand) can be made accordingly. The parameters and decision variables of our GCDES model are introduced in Tables 2 and 3, respectively.

The GCDES decides on the capacity of renewable energy generation and storage components to be built in the area of interest by minimizing the annualized total cost and $\mathrm{CO}_{2}$ emissions. Fixed costs $\left(c_{b}, c_{s}, c_{w}^{i}\right)$ represent the cost of the renewable resource investment, which includes capital, operation, and maintenance costs. We annualize these investment costs by multiplying each component by its annualization factor, which is calculated using the equivalent annual cost formula considering the discount rate $(d r)$ and the respective lifetime of a component. We show an example calculation for solar in Formula 1.

$$
\alpha_{s}=\frac{d r}{1+(1-d r)^{-L_{s}}} .
$$


TABLE 2 Parameters, sets

\begin{tabular}{ll}
$T$ & Time horizon $(t \in\{1 \ldots T\})$ \\
$I$ & Set of wind turbine generator (WTG) types \\
$\Theta$ & Set of scenarios $(\theta \in \Theta)$ \\
$d r$ & Discount rate \\
$c_{b}$ & Investment cost of storage unit, $\$ / \mathrm{kWh}$ \\
$c_{s}$ & Investment cost of solar panel, $\$ / \mathrm{m}^{2}$ \\
$c_{w}^{i}$ & Investment cost of WTG type $i, \$ /$ unit \\
$L_{b}$ & Lifetime of storage unit, years \\
\hline$L_{s}$ & Lifetime of solar panel, years \\
\hline$L_{w}^{i}$ & Lifetime of WTG type $i$, years \\
\hline$L_{F T}$ & Duration of feed-in tariff policy, years \\
$L_{s y s t e m}$ & Duration of the GCDES, years \\
$\alpha_{b}$ & Annualization factor for storage unit \\
$\alpha_{s}$ & Annualization factor for solar panel \\
\hline$\alpha_{w}$ & Annualization factor for wind turbines \\
$\alpha_{p s}$ & Annualization factor for sale price of solar energy \\
\hline$\alpha_{p w}$ & Annualization factor for sale price of wind energy \\
$p^{g}$ & Price of electricity purchased from grid (spot price), $\$ / \mathrm{kWh}$ \\
\hline$p^{s}$ & Elevated sale price of solar energy, $\$ / \mathrm{kWh}$ \\
$p^{w}$ & Elevated sale price of wind energy, $\$ / \mathrm{kWh}$ \\
$d_{t}^{\theta}$ & Local demand in $(t, \theta), \mathrm{kWh}$ \\
$v_{t}^{\theta}$ & Wind speed in $(t, \theta), \mathrm{m} / \mathrm{s}$ \\
$r_{t}^{\theta}$ & solar irradiation in $(t, \theta), \mathrm{kW} / \mathrm{m}^{2}$ \\
$\eta_{s}$ & Overall efficiency of solar panel, $\%$ \\
$\eta_{b}$ & Storage efficiency, $\%$ \\
$\kappa$ & Electricity generation limit multiplier \\
\hline$M$ & Maximum unit time demand, $\mathrm{kWh}$ \\
$\beta$ & CO 2 Equivalent emission by electricity grid, tonne $/ \mathrm{kWh}$ \\
\hline
\end{tabular}

The electricity purchase price $\left(p^{g}\right)$ represents the average retail price of electricity in the market. Governments that practise a feed-in tariff policy offer different elevated sale prices (higher than the retail price of electricity) for each renewable energy resource to investors. ${ }^{18}$ This policy has different prices for each renewable source $\left(p^{w}, p^{s}\right)$ and is usually available for a limited amount of time. Therefore, incentivized prices cannot be used throughout the lifespan of the system. After the feed-in tariff expires, green energy becomes available to the market at retail price. Thus, we distribute the effect of an elevated sale price $\left(p^{s}, p^{w}\right)$ across the lifetime of the system. Formula 2 calculates the annualization factor for the sale price of solar energy $\left(\alpha_{p s}\right)$, where $L_{F T}$ represents the duration of the feed-in tariff policy. The same formula is used to calculate the annualization factor for the sale price of wind energy $\left(\alpha_{p w}\right)$ by replacing $\left(p^{s}\right)$ with $\left(p^{w}\right)$.

$$
\alpha_{p s}=\frac{p^{s} L_{F T}+p^{g}\left(L_{\text {system }}-L_{F T}\right)}{p^{s} L_{\text {system }}} .
$$

TABLE 3 Decision variables

\begin{tabular}{ll}
$A_{b}$ & Size of storage unit, $\mathrm{kWh}$ \\
$A_{s}$ & Size of solar panels, $\mathrm{m}^{2}$ \\
$A_{w}^{i}$ & Number of WTGs of type $i$ \\
$S_{t}^{\theta}$ & Electricity generated by solar panels in $(t, \theta), \mathrm{kWh}$ \\
$S D_{t}^{\theta}$ & Solar electricity used to satisfy demand in $(t, \theta), \mathrm{kWh}$ \\
$S B_{t}^{\theta}$ & Solar electricity used to charge battery in $(t, \theta), \mathrm{kWh}$ \\
$S S_{t}^{\theta}$ & Solar electricity sold to grid in $(t, \theta), \mathrm{kWh}$ \\
$W_{t}^{\theta}$ & Electricity generated by WTGs in $(t, \theta), \mathrm{kWh}$ \\
$W D_{t}^{\theta}$ & Wind electricity used to satisfy demand in $(t, \theta), \mathrm{kWh}$ \\
$W B_{t}^{\theta}$ & Wind electricity sent to storage in $(t, \theta), \mathrm{kWh}$ \\
$W S_{t}^{\theta}$ & Wind electricity sold to grid in $(t, \theta), \mathrm{kWh}$ \\
$B_{t}^{\theta}$ & State of charge at the end of time t in scenario $\theta, \mathrm{kWh}$ \\
$B D_{t}^{\theta}$ & Discharge amount in $(t, \theta), \mathrm{kWh}$ \\
$G_{t}^{\theta}$ & Amount of electricity supplied from the grid in $(t, \theta), \mathrm{kWh}$ \\
\hline$X_{t}^{\theta}$ & 1, if electricity is not purchased from the grid in $(t, \theta)$ \\
& 0, if electricity is not fed to the grid in $(t, \theta)$ \\
\hline
\end{tabular}

\subsection{1 | Mathematical model formulation}

$$
\begin{gathered}
\min \mathrm{Z} 1: \alpha_{b} c_{b} A_{b}+\alpha_{s} c_{s} A_{s}+\alpha_{w} \sum_{i \in I} c_{w}^{i} A_{w}^{i} \\
+\frac{1}{|\Theta|} \sum_{\theta \in \Theta} \sum_{t \in T}\left[p^{g} G_{t}^{\theta}-\alpha_{p s} p^{s} S S_{t}^{\theta}-\alpha_{p w} p^{w} W S_{t}^{\theta}\right] \\
\min \mathrm{Z} 2: \beta \frac{1}{|\Theta|} \sum_{\theta \in \Theta} \sum_{t \in T} G_{t}^{\theta}
\end{gathered}
$$

s.t

$$
S_{t}^{\theta}=\eta_{s} r_{t}^{\theta} A_{s} \quad \forall t \in\{1 \ldots T\}, \quad \forall \theta \in \Theta
$$

$$
W_{t}^{\theta}=\sum_{i \in I} f^{i}\left(v_{t}^{\theta}\right) A_{w}^{i} \forall t \in\{1 \ldots T\}, \quad \forall \theta \in \Theta
$$

$$
S_{t}^{\theta}=S S_{t}^{\theta}+S D_{t}^{\theta}+S B_{t}^{\theta} \quad \forall t \in\{1 \ldots T\}, \quad \forall \theta \in \Theta
$$

$W_{t}^{\theta}=W S_{t}^{\theta}+W D_{t}^{\theta}+W B_{t}^{\theta} \quad \forall t \in\{1 \ldots T\}, \quad \forall \theta \in \Theta$

$d_{t}^{\theta}=S D_{t}^{\theta}+W D_{t}^{\theta}+\eta_{b} B D_{t}^{\theta}+G_{t}^{\theta} \quad \forall t \in\{1 \ldots T\}, \quad \forall \theta \in \Theta$

$B_{t}^{\theta}=B_{t-1}^{\theta}+S B_{t}^{\theta}+W B_{t}^{\theta}-B D_{t}^{\theta} \quad \forall t \in\{1 \ldots T\}, \quad \forall \theta \in \Theta$

$$
\kappa M \geqslant S_{t}^{\theta}+W_{t}^{\theta} \quad \forall t \in\{1 \ldots T\}, \forall \theta \in \Theta
$$

$$
\kappa M X_{t}^{\theta} \geqslant S S_{t}^{\theta}+W S_{t}^{\theta} \quad \forall t \in\{1 \ldots T\}, \quad \forall \theta \in \Theta
$$




$$
\begin{aligned}
& |T| M X_{t}^{\theta} \geqslant S B_{t}^{\theta}+W B_{t}^{\theta} \quad \forall t \in\{1 \ldots T\}, \quad \forall \theta \in \Theta \\
& M\left(1-X_{t}^{\theta}\right) \geqslant B D_{t}^{\theta} \quad \forall t \in\{1 \ldots T\}, \forall \theta \in \Theta \\
& M\left(1-X_{t}^{\theta}\right) \geqslant G_{t}^{\theta} \\
& \forall t \in\{1 \ldots T\}, \quad \forall \theta \in \Theta \\
& A_{b} \geqslant B_{t}^{\theta} \\
& \forall t \in\{1 \ldots T\}, \quad \forall \theta \in \Theta \\
& B_{0}^{\theta}=0 \\
& B_{T}^{\theta}=0 \\
& S_{t}^{\theta}, B_{t}^{\theta}, W_{t}^{\theta}, G_{t}^{\theta} \geqslant 0 \\
& \forall t \in\{1 \ldots T\}, \quad \forall \theta \in \Theta \\
& S B_{t}^{\theta}, S S_{t}^{\theta}, W S_{t}^{\theta}, W B_{t}^{\theta} \geqslant 0 \\
& \forall t \in\{1 \ldots T\}, \quad \forall \theta \in \Theta \\
& S D_{t}^{\theta}, W D_{t}^{\theta}, B D_{t}^{\theta} \geqslant 0 \\
& \forall t \in\{1 \ldots T\}, \quad \forall \theta \in \Theta \\
& A_{s}, A_{b}, A_{w}^{i} \geqslant 0 \\
& A_{w}^{i} \in \mathbb{Z}_{\geqslant 0}, \forall i \in I \\
& X_{t}^{\theta} \in\{0,1\} \\
& \forall t \in\{1 \ldots T\}, \quad \forall \theta \in \Theta
\end{aligned}
$$

In our mathematical model, we have 2 objective functions: $\mathrm{Z} 1$ and $\mathrm{Z} 2$. The first objective represents the summation of the total investment and expected operational costs, which correspond to the first- and second-stage decisions, respectively. The second objective function, $\mathrm{Z2}$, is for the $\mathrm{CO}_{2}$-equivalent emissions amount, which can be calculated using different forms of functions of the electricity purchased from the grid. In this setting, a linear function is used with the rate of emission $(\beta)$, which depends on the proportion of fossil fuel-based electricity in the grid network and increases as the proportion of the fossil fuel increases.

For each scenario $\theta$ and time unit $t$, generated solar and wind energy are calculated in constraints 5 and 6 , respectively. In constraint 6 , the wind energy output in time $t$ in scenario $\theta$ is calculated using $f^{i}$, the piecewise linear function of the WTG type $i$. Constraints 7 and 8 are used to represent the distribution of generated energy, which can be used to meet local demand, sold directly to the grid or stored. Constraint 9 guarantees that demand is met in each time unit and scenario by generated renewable energy, energy in the storage device, or electricity from the grid. Energy production is limited with a bound, based on the physical limitations of the area. With constraint 11, total energy production within a unit time is limited by $\kappa M$, where $M$ can be considered as a very big number and $\kappa$ is a constant multiplier. For this study, $M$ is taken as the amount of peak demand observed during the planning horizon. By changing $\kappa$, the dependency of optimal sizes on the physical limitations can be investigated.

The binary variable $X_{t}^{\theta}$ is used in constraints 12 to 15 to ensure that local demand has priority over storage and selling; that is, only excess energy can be sold or stored. In our setting, we use storage and the grid network as backup components, which can be used to satisfy demand only in case of an energy deficit. The constraints 12 to 15 guarantee that generated renewable energy will be used first to satisfy local demand.

In constraint 16 , it is ensured that state of charge at time unit $t$ cannot exceed the nominal capacity of the storage unit. It is assumed that the storage is empty at the beginning and at the end of the horizon, which is ensured by constraints 17 and 18. The nonnegativity of variables is satisfied with 19 to 22 .

The nondominated solutions of the GCDES model are found using the $\varepsilon$-constraint method, which is widely used for bi-objective problems. ${ }^{20}$ The method is based on solving single-objective models iteratively, limiting the second objective function value by a constraint. Note that to guarantee that the solutions are nondominated in the strict sense, one must ensure that for each level of the objective that is optimized, the solution that provides the best value in terms of the other objective should be returned. In other words, in our setting, among alternative solutions with the same total cost value, the solution that gives the minimum $\mathrm{CO}_{2}$ emission level should be found. This result can be ensured by solving 2 models at each iteration of the $\varepsilon$-constraint method; first, we solve a model that minimizes cost to obtain the optimal cost value, and then we solve a model in which $\mathrm{CO}_{2}$ emissions are minimized over solutions that have this cost value. From now on, we refer to the approach based on solving the GCDES model with the $\varepsilon$-constraint method as the GCDES approach. The schematic description of this approach can be found in Figure 2 .

\subsection{Simulation-optimization approach}

Our problem has a multi-stage stochastic nature because of the intermittency of renewable resources. A 2-stage 


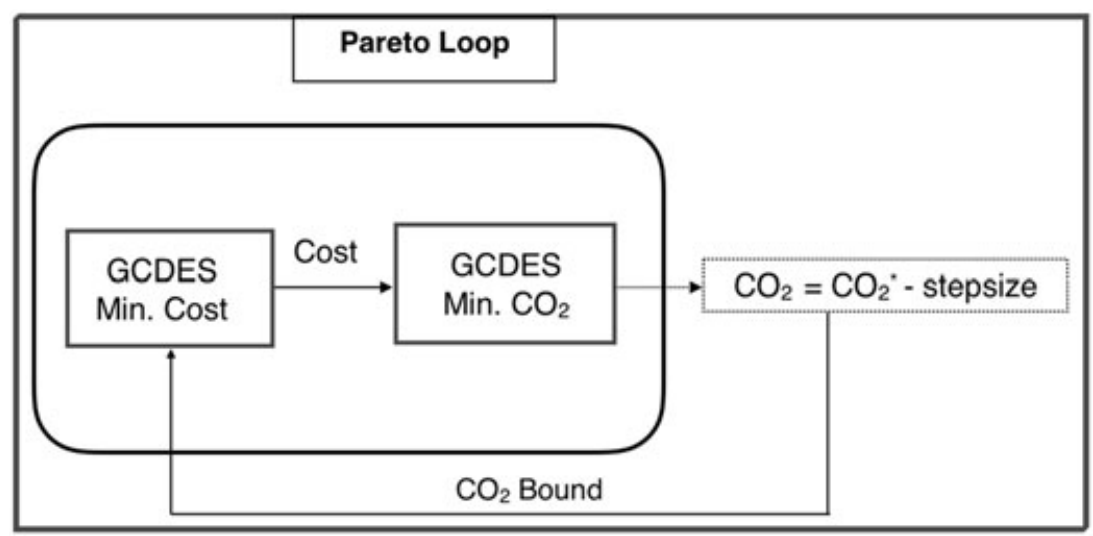

FIGURE 2 Grid-connected decentralized energy system (GCDES) approach

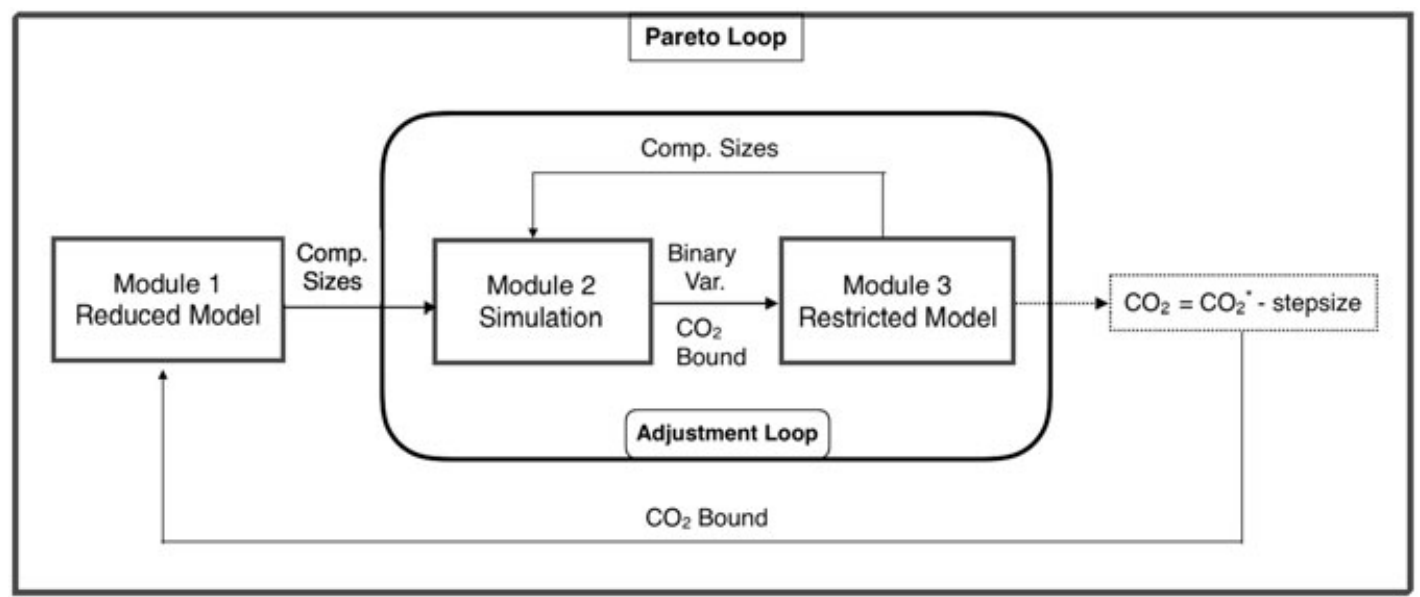

FIGURE 3 Simulation-optimization approach

stochastic programming model partially handles this uncertainty by generating scenarios and making decisions such that the expected value of a function over all scenarios is optimized. Such a model, however, still violates nonanticipativity constraints; that is, it makes operational decisions assuming that the availability pattern reflected in each scenario is known in advance (eg, knowing what the hourly wind speed will be for the whole planning horizon), which is not the case in real life. In reality, operators observe the availability of renewables in a time period and make operational decisions accordingly, following a given policy. With the hope of addressing this multi-stage decision-making process in a computationally tractable way, we introduce a simulation-optimization (SO) approach, which includes 2 different versions of the GCDES model and a simulation module, where the simulation module handles nonanticipativity issues.

The flow diagram of the SO approach we propose can be seen in Figure 3. The overall algorithm works as a variant of the $\varepsilon$-constraint method.

\subsection{1 | Module 1-reduced version of the GCDES model}

Our algorithm starts with solving the reduced version of the GCDES model. The main reason for using the reduced version is to obtain the initial component sizes to be fed into the simulation module. In this version, constraints 14 and 15 , which include the binary variable $X_{t}^{\theta}$, are relaxed to reduce computational effort. This method enables the module to obtain the optimal component sizes of a setting in which renewable energy can be sold to the grid while meeting demand using electricity from the grid. The results of this model (the number of wind turbines, the solar panel area, and the storage size) are used as inputs for the simulation module.

\subsection{2 | Module 2-simulation}

Taking the component sizes (first-stage decision variables) as inputs in the simulation module, the operator follows a policy to make operational decisions (second-stage decision variables) without knowledge of the future availability 
of wind and solar resources. The policy is designed to prioritize renewable sources while meeting local demand, which is in line with the objective of minimizing the $\mathrm{CO}_{2}$ emission value. This module takes the investment decisions (the number of wind turbines, the solar panel area, and the storage size) obtained from the reduced version of the GCDES model and calculates the renewable energy generated at each time period of the planning horizon for each scenario. First, local demand is satisfied using a less-profitable renewable energy source, and then excess energy is transferred to the storage unit until it is full. If there is still excess energy, it is sold to the grid. When there is insufficient renewable energy to satisfy local demand, first, the amount is discharged from the storage unit if possible and then any deficit amount is purchased from the grid. In this way, the simulation module determines whether to sell renewable energy or outsource fossil fuel-based electricity from the grid, which corresponds to the binary variables in the GCDES model $\left(X_{t}^{\theta}\right)$. The resulting total $\mathrm{CO}_{2}$ emission value and related binary variables $\left(X_{t}^{\theta}\right)$ are used as inputs in module 3 (the restricted version of the GCDES model).

\subsection{3 | Module 3-restricted version of the GCDES model}

In the restricted version, the output of the simulation module is used to dictate purchasing/selling decisions. These decisions are conveyed to the model by fixing binary variables $\left(X_{t}^{\theta}\right)$ in constraints 14 and 15 . Moreover, the total $\mathrm{CO}_{2}$ emission value observed in the simulation module is used to update the $\mathrm{CO}_{2}$ limit in the restricted model. This model is solved, and the new investment decisions are fed back to the simulation module, which now applies the policy using the new component sizes. In this way, the component sizes and the purchasing/selling decisions can be adjusted iteratively. This adjustment continues until the decisions made in modules 2 and 3 are in line with each other, and the (adjustment) loop terminates when the improvement in cost is less than $0.1 \%$. Each such loop provides a solution with a corresponding cost and $\mathrm{CO}_{2}$ level. To move to the next (neighbor) solution, we further restrict the $\mathrm{CO}_{2}$ limit by subtracting a predetermined amount (step size) from the $\mathrm{CO}_{2}$ level of the latest solution found. A new (Pareto) loop is initiated by solving module 1 with this new $\mathrm{CO}_{2}$ limit (see the outer loop in Figure 3).

\subsection{Metaheuristic approaches}

For the addressed problem, we present metaheuristic algorithms that consist of 2 modules as in the SO approach. In the optimization module, existing multi-objective metaheuristic algorithms are used to obtain a Pareto front and the simulation module is the same with that of the SO approach discussed in Section 4.2. These procedures start with an initial set of solutions with randomly generated component sizes. Then these solutions are conveyed to the module 2 so as to perform the simulation. Given an operation policy and the component sizes, the simulation module makes the operational decisions and the objective function values are calculated. The resulting cost and $\mathrm{CO}_{2}$ emission values are returned to the optimization module. Following the algorithm steps, an approximate Pareto front is generated in the end. The working scheme of the approach can be found in Figure 4.

As seen in the Figure 4, simulation and optimization modules operate in a loop where they provide input for each other. For the simulation purposes, we resort to the previously discussed module 2, while optimized MOPSO (OMOPSO), nondominated sorting genetic algorithm II (NSGA-II), and strength Pareto evolutionary algorithm (SPEA) 2 are employed in the module 1 regarding the optimization.

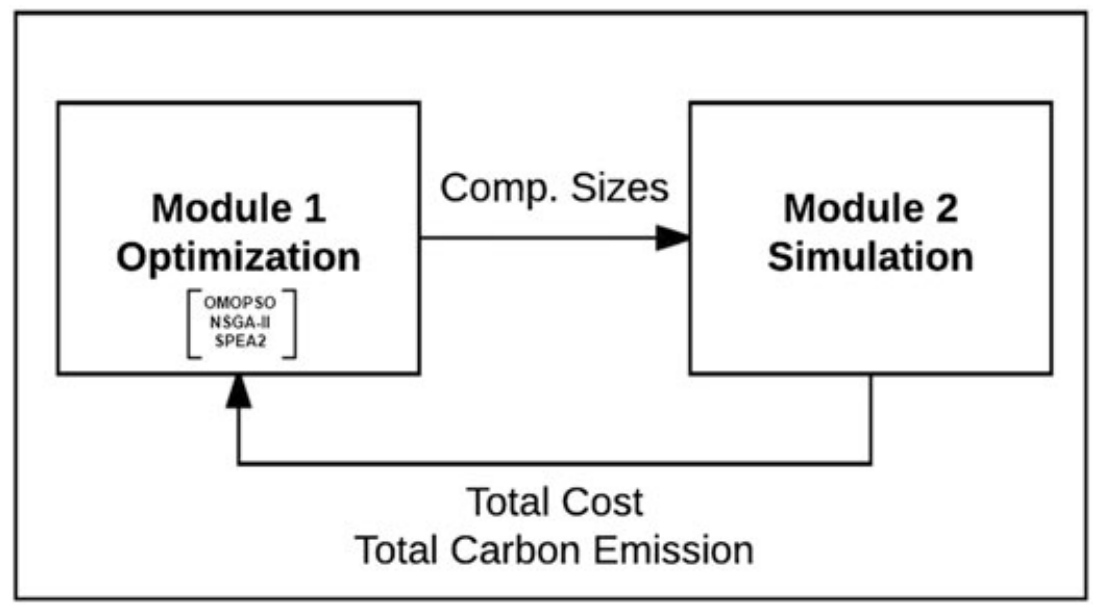

FIGURE 4 Metaheuristic approach 


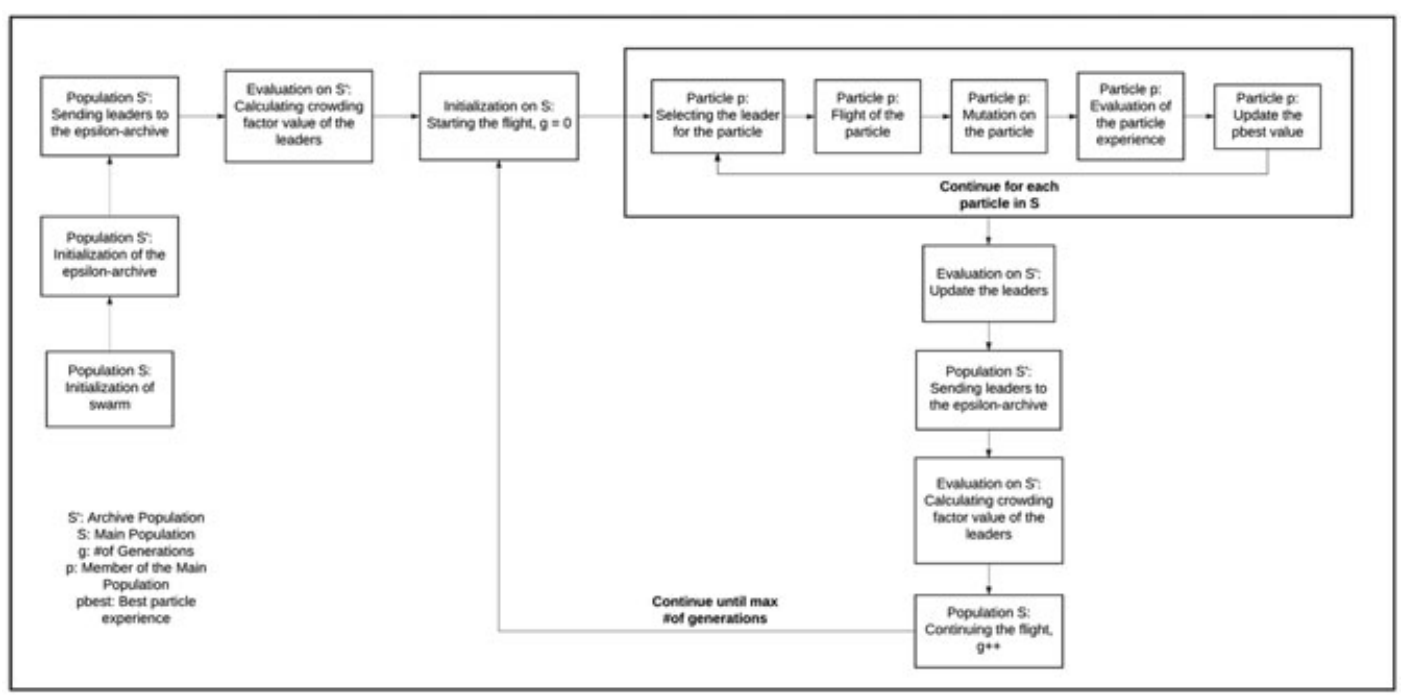

FIGURE 5 Flowchart of the optimized multi-objective particle swarm optimization algorithm

\subsection{1 | Optimized MOPSO}

Particle swarm optimization (PSO) algorithm proposed by Kennedy and Eberhart ${ }^{21}$ is one of the popular metaheuristic algorithms used in diverse optimization tasks. This algorithm mimics the flocking of birds and tries to find an optimal solution throughout the search space. At the initial step of the procedure, a set of solutions called swarms or particles is generated. As these swarms fly over the feasible area, each particle updates its velocity and position according to a group of equations. These equations consider both the best solution that particle has found so far and all the best solutions found by the members of the swarm. In the end, the optimal solution is attained via particle experience and best global particle. ${ }^{21}$

Although PSO performs well in a range of optimization problems, its application is restricted to the single objective problems. ${ }^{22}$ Extending the idea of PSO to multi-objective problems, Coello and Lechuga ${ }^{22}$ develop MOPSO algorithm. The main idea of MOPSO is including a global repository in which each particle deposits its flight experience after a cycle. Later, this repository is used by the swarms to select the leader that guides the flight. Differently from the PSO, the result is a set of nondominated solutions that are kept in the repository. ${ }^{22}$

Many algorithms originated from MOPSO can be found in the literature. ${ }^{23}$ Although they have the common idea of selecting the leader from the nondominated particles, the selection process could be different. To compare these MOPSOs, an experimental study is conducted by Durillo et al. ${ }^{23}$ Three benchmark problems and performance metrics are employed so as to make the comparison. The results of the experiments indicate that OMOPSO performs best in all of the studied problems. Because of its superior performance, OMOPSO algorithm is applied to the present problem, minimizing total cost and $\mathrm{CO}_{2}$ emission simultaneously. The algorithm runs following the steps outlined in Figure 5.

\subsection{2 | Nondominated sorting genetic algorithm II}

Genetic algorithms are used for solving a variety of multiobjective optimization problems, thanks to their ability to provide good solutions in a reasonable amount of time. ${ }^{20}$ These algorithms work according to the evolutionary principles in the optimization of both continuous and discrete valued problems. Nondominated sorting genetic algorithm II, one of the genetic algorithms, has been applied to different multi-objective optimization tasks because of its convergence performance. ${ }^{24}$ The procedure starts with randomly generating $N$ members. After the generation, these members are sorted into different nondomination levels by pairwise comparisons. When the sorting task is completed, each member is assigned 2 attributes, which are later used in the selection. Having these properties, the members experience selection and mutation, which cause changes in the member itself. ${ }^{24}$ In the end, the offspring population of size $N$ is obtained. To store the solutions from the parent population, former and offspring populations are combined. The recently created population with $2 N$ members is nondomination sorted. There should be a selection between these nondomination sorted members so as to maintain the size $N$. In the selection, previously assigned properties are taken into account. This whole process continues until a predefined number of generations are reached. At the end of all steps, a Pareto set of solutions is obtained. ${ }^{24}$ Generational loop of the NSGA-II can be found in Figure 6. 


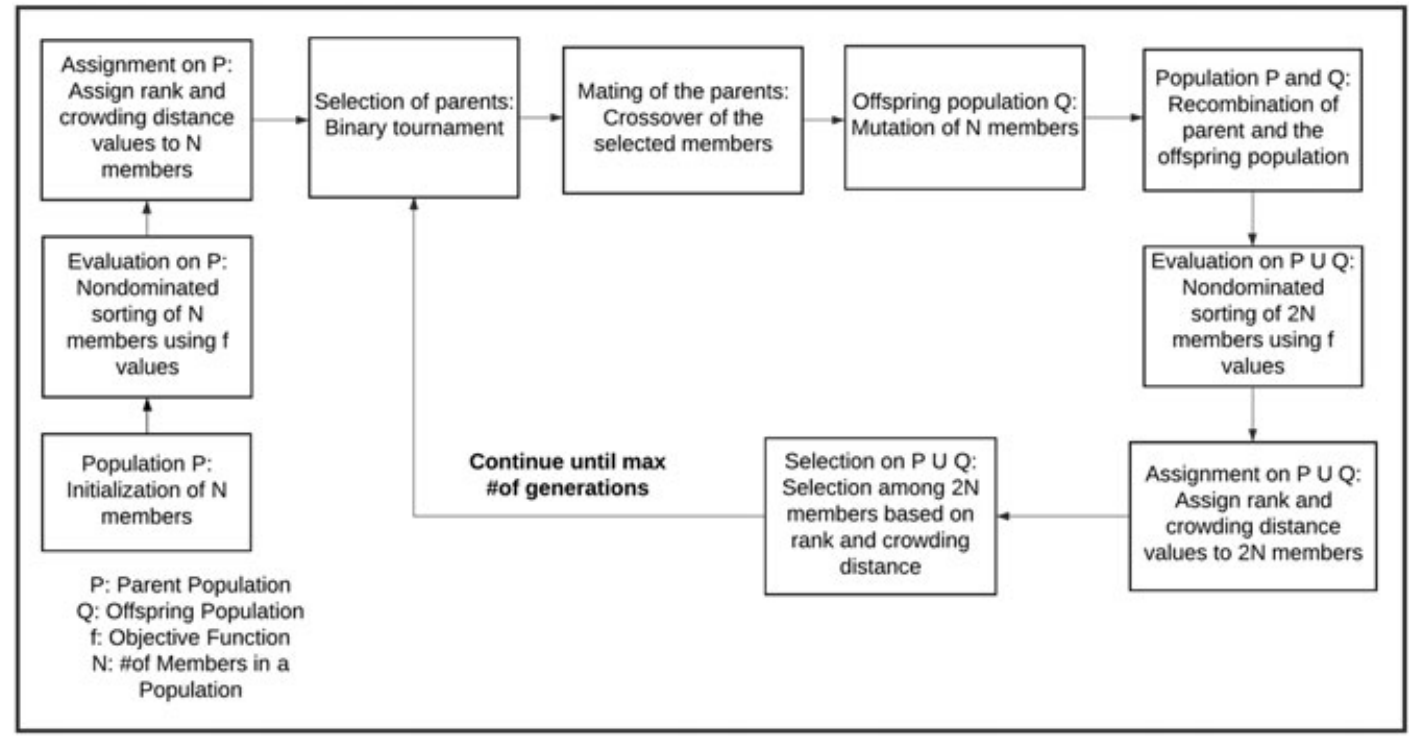

FIGURE 6 Flowchart of the nondominated sorting genetic algorithm II

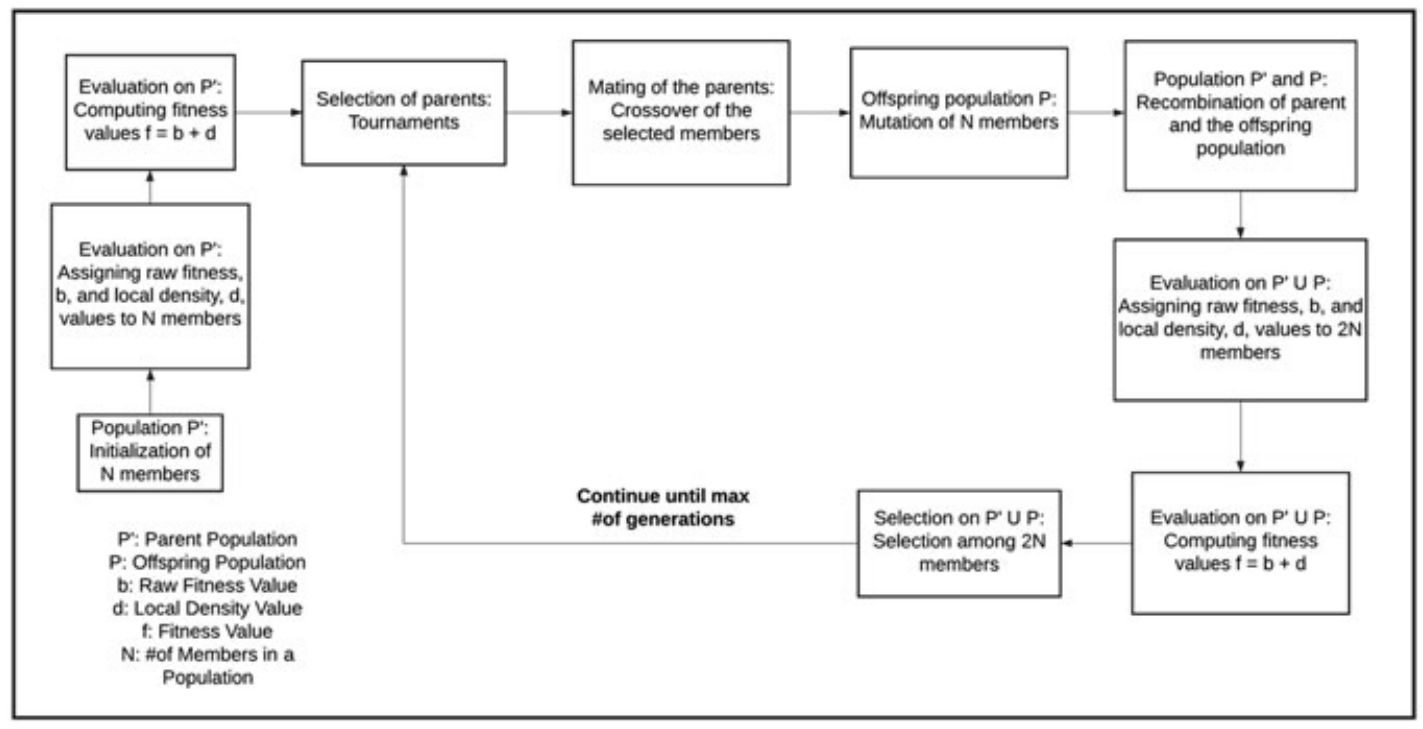

FIGURE 7 Flowchart of the strength Pareto evolutionary algorithm 2

\subsection{3 | Strength Pareto evolutionary algorithm 2}

Strength Pareto evolutionary algorithm 2 is another evolutionary multi-objective optimization technique that could approximate the set of optimal solutions in a single optimization run. ${ }^{20}$ The algorithm is introduced by Zitzler, Laumanns, and Thiele ${ }^{25}$ so as to eliminate the potential weaknesses of its predecessor SPEA. The method is initialized by creating a population and an external archive. Following the initialization, the members both in population and archive are assigned fitness values and they are selected using these values with a tournament to fill the mating pool. The selected parents mate with each other and the resulting offspring are exposed to mutation. Because the algorithm takes the parent population into consideration while searching for the solution, the old and offspring populations are brought together. The evolution process continues until the maximum number of iterations is attained. When the process reaches the predefined number of iterations, the set of optimal solutions becomes available. ${ }^{25}$ See Figure 7 for the flowchart.

\section{5 | NUMERICAL STUDIES}

\section{1 | Data generation}

We use different data sets to analyze the effect of location differences. For this purpose, 3 different levels of resource availability are determined (high, medium, and low) for 
TABLE 4 Renewable resource availability data

\begin{tabular}{lllllll} 
& \multicolumn{3}{l}{ Wind speed $(\mathbf{m} / \mathbf{s})$} & \multicolumn{3}{c}{ Solar irradiation $\left(\mathbf{k W} / \mathbf{m}^{2}\right)$} \\
\hline Data Set & Min & Mean & Max & Min & Mean & Max \\
High & 0.21 & 7.81 & 29.90 & 0 & 0.24 & 1.09 \\
Medium & 0.13 & 5.14 & 19.35 & 0 & 0.17 & 0.97 \\
Low & 0.02 & 3.33 & 11.92 & 0 & 0.08 & 0.66
\end{tabular}
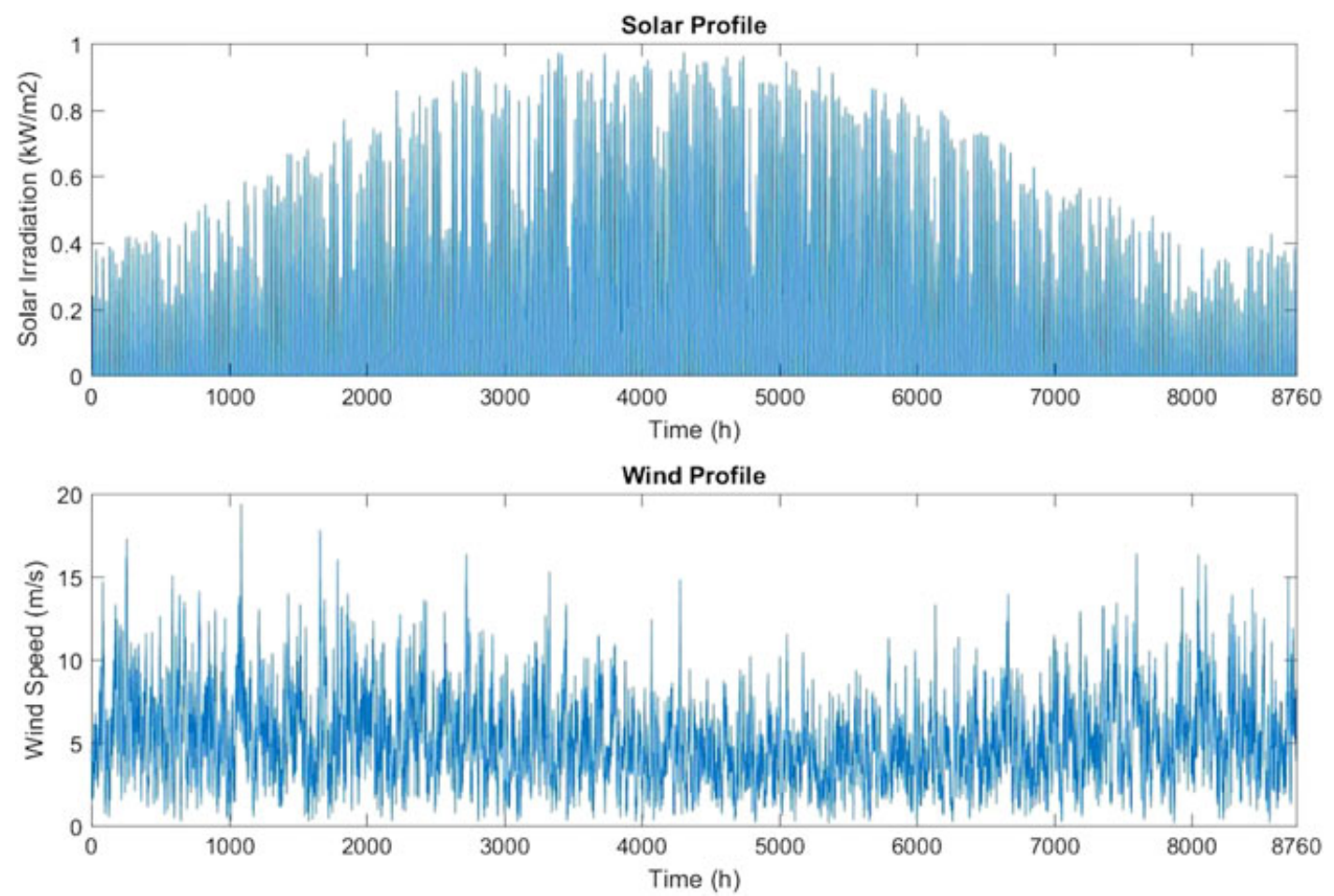

FIGURE 8 Solar and wind profiles for medium availability level [Colour figure can be viewed at wileyonlinelibrary.com]

solar and wind energy alike. Solar irradiation and wind speed data are gathered using Hybrid Optimization of Multiple Energy Resources software. Statistics for the different levels of resource availability data can be found in Table 4 . For example, we represent a place with medium wind speed and medium solar irradiation by generating data with a mean wind speed value of $5.14 \mathrm{~m} / \mathrm{s}$ and mean solar irradiation value of $0.17 \mathrm{~kW} / \mathrm{m}^{2}$.

To illustrate, wind speed and solar irradiation for 1-year profiles for the medium availability level are represented in Figure 8.

For our numerical study, we consider a medium-scale demand point such as a university campus. To generate an illustrative data set, we obtain 1 month of the hourly average electricity consumption data of Bilkent University campus in Turkey. By preserving the electricity consumption characteristics of Bilkent University, hourly consumption profiles for 1 year are generated using Hybrid Optimization of Multiple Energy Resources software. Bilkent's average hourly and monthly electricity consumption can be found in Figure 9.
Three different wind turbine types with differently rated powers are used in the analysis. These turbines have capacities of $0.9,2$, and $3 \mathrm{MW}$. Wind energy generation calculations are made based on the respective power curve of each turbine. ${ }^{26}$ Parameters for the numerical analysis are provided in Table 5. For more information about the parameters and numerical analysis, see Altıntaş. ${ }^{27}$ The GCDES and SO approaches are implemented in MATLAB 9.0 and solved using CPLEX 12.6. The source codes of metaheuristic algorithms written in JAVA environment are executed. ${ }^{28}$ All of these solution procedures are run in a computer with Intel Xeon CPU E5-1650 3.6 GHz processor and 32 GB RAM. The resulting times are expressed in central processing unit (CPU) seconds.

\section{2 | Comparison of metaheuristic algorithms}

So as to assess the quality of Pareto fronts obtained by metaheuristics, performance metrics are introduced in multi-objective optimization literature. These measures 

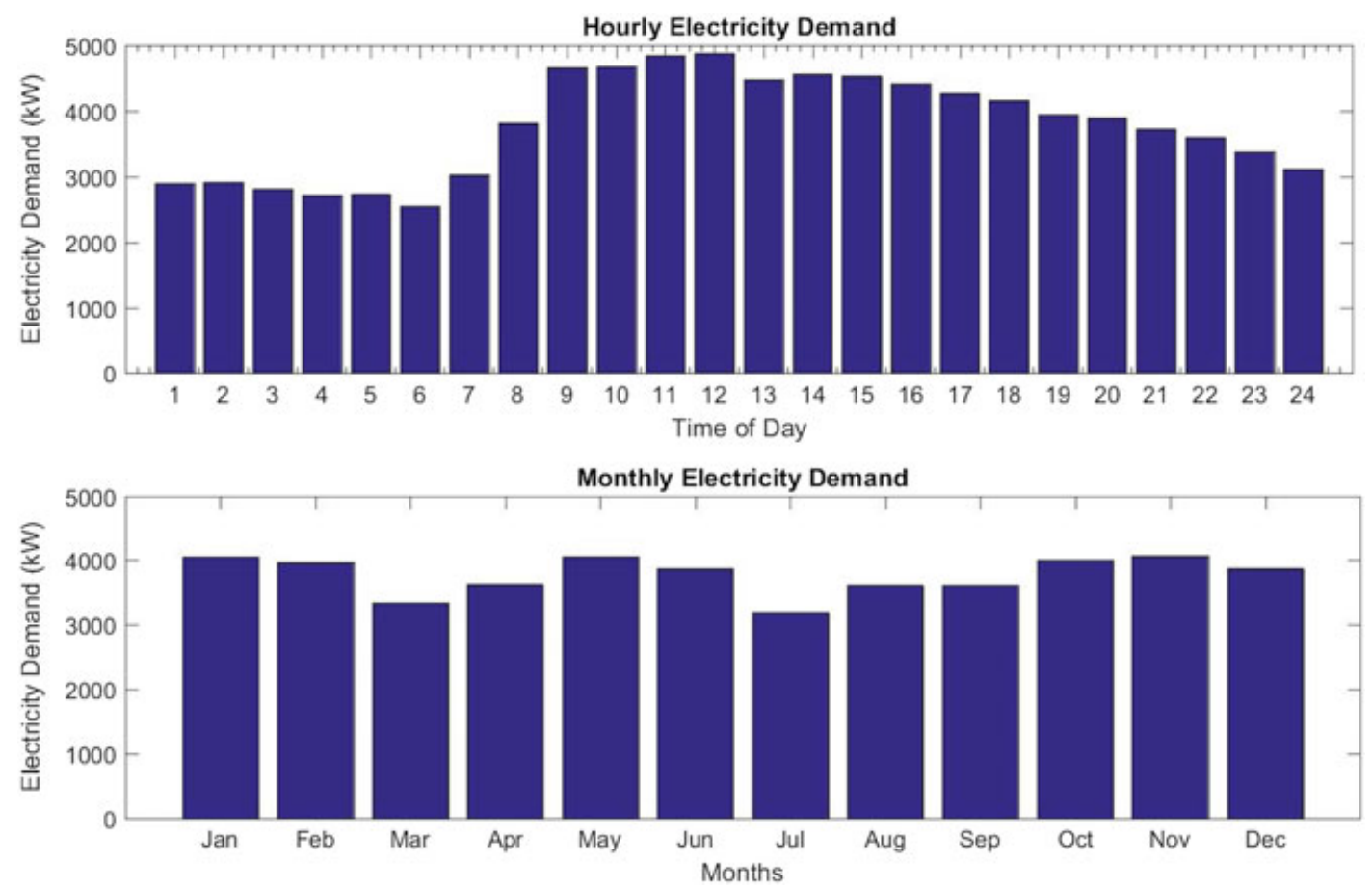

FIGURE 9 Hourly and monthly averages of campus demand [Colour figure can be viewed at wileyonlinelibrary.com]

TABLE 5 Parameters for numerical study

\begin{tabular}{llll}
$c_{b}$ & $\$ 330 / \mathrm{kWh}$ & $p^{g}$ & $\$ 0.06 / \mathrm{kWh}$ \\
$c_{s}$ & $\$ 300 / \mathrm{m}^{2}$ & $p^{s}$ & $\$ 0.13 / \mathrm{kWh}$ \\
$c_{w}^{900} \quad \mathrm{~kW}$ & $\$ 1.77 \mathrm{M}$ & $p^{w}$ & $\$ 0.07 / \mathrm{kWh}$ \\
$c_{w}^{2} \quad \mathrm{MW}$ & $\$ 4.3 \mathrm{M}$ & $\eta_{s}$ & 12 \\
$c_{w}^{3} \quad \mathrm{MW}$ & $\$ 5.49 \mathrm{M}$ & $\eta_{b}$ & 80 \\
$L_{b}$ & 10 years & $r$ & 0.05 \\
$L_{s}$ & 30 years & $\kappa$ & 2 \\
$L_{w}$ & 20 years & $\beta$ & 0.0004836 \\
$L_{s y s t e m}$ & 30 years & $T$ & 8760 hours \\
$L_{F T}$ & 10 years & & \\
\hline
\end{tabular}

can be categorized into 3 groups based on their ability to depict a certain aspect of the solution set. ${ }^{29}$

1. Metrics that assess convergence to the known Pareto-optimal front.

2. Metrics that evaluate spread of the solutions on the Pareto-optimal front.

3. Metrics that measure combinations of solutions' convergence and spread.

In this study, each Pareto front is evaluated using various metrics from the above categories. Because the true Pareto front is unknown, it is not possible to measure the convergence truly. Instead, coverage value is computed so as to compare 2 Pareto sets with each other. Together with the coverage metric, hypervolume measure from the third category is employed to assess the convergence and spread of solutions simultaneously. In addition to this assessment,

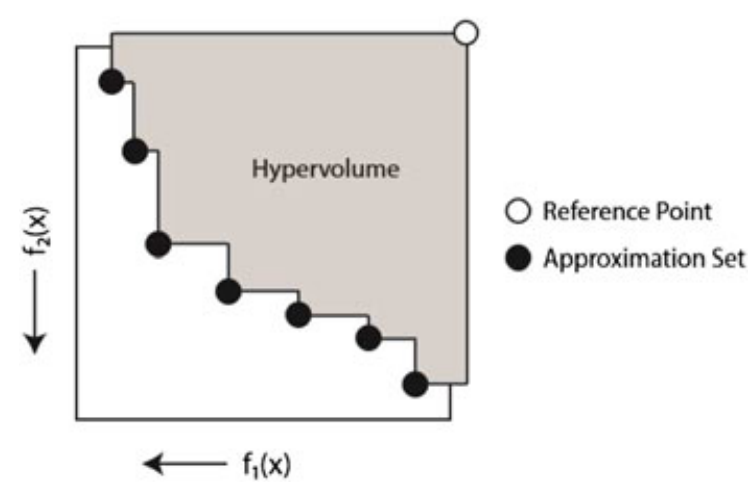

FIGURE 10 Hypervolume measure for a 2-objective minimization case [Colour figure can be viewed at wileyonlinelibrary.com]

spacing and maximum spread values are calculated for evaluating the solutions' spread. 
Spacing (S): The measure is proposed by $\mathrm{Schott}^{30}$ and has been used to estimate the diversity of Pareto front. In the formulation, $n$ stands for the number of solutions in frontier, $d_{i}$ represents the minimum Manhattan distance between a solution $i$ and any other solution, and $\bar{d}$ is the average distance between 2 solutions. As $\mathrm{S}$ becomes zero, a more uniformly distributed Pareto front is attained.

$$
S=\sqrt{\frac{1}{n-1} \times \sum_{i=1}^{n}\left(d_{i}-\bar{d}\right)^{2}}
$$

Maximum spread (MS): Zitzler ${ }^{31}$ introduces maximum spread metric to measure the spread of a given set. For this measure, greater values are preferred because they indicate a better spread of the points. The measure is calculated as follows where $a_{i}$ and $b_{i}$ are 2 solutions in Pareto frontier and $n$ is the number of solutions.

$$
\mathrm{MS}=\sqrt{\sum_{i=1}^{n} \max \left(\left\|a_{i}-b_{i}\right\|\right)} .
$$

Coverage (C): The coverage metric, suggested by Zitzler, ${ }^{31}$ is used to determine whether a Pareto front dominates another. So as to make the comparison between 2 Pareto fronts, $A$ and $B$, each solution from one front is compared with all solutions in the other front. Two points, $a$ and $b$, are compared with each other at a time using the weakly dominance operator shown as $\geq$. The coverage values of sets $A$ and $B$ are represented by $C(A, B)$ and $C(B, A)$. If $C(A, B)$ is equal to 1 , it means all solutions in the set $B$ are weakly dominated by $A$. In the opposite case, where $C(A, B)$ is 0 , none of the points in $B$ is weakly dominated by $A$. Because there could be interaction between 2 sets, both directions should be considered in the calculation.

$$
\mathrm{C}(\mathrm{A}, \mathrm{B})=\frac{|\{b \in B \mid \exists a \in A: a \geq b\}|}{|B|} .
$$

Hypervolume: The hypervolume measure is first proposed by Zitzler and Thiele ${ }^{32}$ as the size of space covered. With change in the name over the time, this metric has been applied for evaluating the Pareto solutions' convergence and spread at the same time. For this purpose, the hypervolume quantifies the volume of the dominated space, which is enclosed with a reference point. Usually, the point in the reference set having the worst-case results for each of the objective is selected, and by adding some delta value, the reference point is reached. Considering a 2-objective minimization problem setting, with objective functions $f_{1}(x)$ and $f_{2}(x)$, the computed area can be seen in Figure $10 .{ }^{33}$

Given the economic parameters and the data sets, 3 well-known algorithms, OMOPSO, NSGA-II, and SPEA2, are tested for a medium solar-medium wind problem instance with 9 scenarios. To find the parameter configuration of each algorithm, different generations are tried. By changing the generation count, various sets of optimal solutions are obtained. These solutions are compared based on some popular performance metrics, and their values are recorded in Tables 6 to 8 . So as to provide an example for the tuning of the algorithms, OMOPSO algorithm is selected, and its generation count is arranged based on the metric results in Table 6 .

All of the performance metric results indicate that 5000 and 10000 evaluations should be preferred over the others. Provided that there is no significant difference between 5000 and 10000 evaluations in terms of the other performance measures, the version, which takes less CPU time, 5000 is chosen. Tunings of the other algorithms are completed following the same steps here and in the end 5000 generations is accepted as the parameter of the 3 algorithms.

Using 5000 generations, statistical analyses are conducted. For this purpose, each metaheuristic algorithm is run for 10 times and their results are compared based on the hypervolume measure. This metric is selected because it evaluates both convergence and spread of the solutions. Minimum, mean and maximum values of this measure are listed in Table 9. To understand which algorithm performs the best, hypothesis testing is conducted. While checking if there is a significant difference between OMOPSO, NSGA-II, and SPEA2, Kruskal-Wallis and Mann-Whitney $U$ tests are applied. ${ }^{34}$ The results of these tests clearly show that OMOPSO is the best performing algorithm for the given problem. When the other 2 algorithms are compared with each other, no significant difference is found. Therefore, OMOPSO algorithm is used for obtaining the set of Pareto solutions in our stochastic bi-objective optimization of the GC system.

\begin{tabular}{|c|c|c|c|c|}
\hline Performance metric/\# of evaluations & 500 & 1000 & 5000 & 10000 \\
\hline Spacing & 0.0154 & 0.0047 & 0.0009 & 0.0005 \\
\hline Max Spread & 9.5723 & 14.2817 & 35.1018 & 49.8013 \\
\hline CPU Time, s & 143.95 & 289.08 & 1367.16 & 2818.88 \\
\hline
\end{tabular}

TABLE 6 Performance measure results for optimized multi-objective particle swarm optimization algorithm 
TABLE 7 Performance measure results for strength Pareto evolutionary algorithm 2 algorithm

\begin{tabular}{lcccc} 
Performance metric/\# of evaluations & \multicolumn{1}{c}{$\mathbf{5 0 0}$} & $\mathbf{1 0 0 0}$ & $\mathbf{5 0 0 0}$ & $\mathbf{1 0 ~ 0 0 0}$ \\
\hline Spacing & 0.0120 & 0.0128 & 0.0039 & 0.0042 \\
Max Spread & 10.4554 & 10.1637 & 10.2725 & 10.2664 \\
CPU Time (s) & 141.44 & 290.30 & 1423.75 & 2930.03 \\
\hline
\end{tabular}

TABLE 8 Performance measure results for nondominated sorting genetic algorithm II algorithm

\begin{tabular}{lcccc} 
Performance metric/\# of evaluations & $\mathbf{5 0 0}$ & $\mathbf{1 0 0 0}$ & $\mathbf{5 0 0 0}$ & $\mathbf{1 0 ~ 0 0 0}$ \\
\hline Spacing & 0.0122 & 0.0074 & 0.0072 & 0.0069 \\
Max Spread & 9.3381 & 10.1065 & 10.0013 & 10.2081 \\
CPU Time (s) & 144.83 & 279.61 & 1406.53 & 2935.09 \\
\hline
\end{tabular}

TABLE 9 Hypervolume metric results for algorithms

\begin{tabular}{llllll} 
Algorithm & Hypervolume & & Count & Indifferent \\
\hline & Min & Median & Max & & \\
OMOPSO & 0.579 & 0.581 & 0.582 & 10 & - \\
NSGA2 & 0.571 & 0.576 & 0.578 & 10 & SPEA2 \\
SPEA2 & 0.570 & 0.578 & 0.579 & 10 & NSGA2 \\
\hline
\end{tabular}

TABLE 10 Attributes of generated scenarios

\begin{tabular}{lllllllllll}
\multicolumn{7}{c}{ Solar irradiation $\left(\mathbf{k W} / \mathbf{m}^{2}\right)$} \\
\cline { 2 - 12 }
\end{tabular}

\section{3 | Comparative analysis}

In this part, 3 solution methods (GCDES, SO, and OMOPSO) are compared with each other by taking the stochasticity of a location's renewable resources into consideration. To handle the stochasticity aspect of the problem, a scenario-wise approach is used. For solar data, scenarios are formed via perturbation of the profiles shown in Figure 8. Choosing the parameter as 5\%, the procedure of Kuznia et $\mathrm{al}^{7}$ is followed in the scenario generation. For wind data, techniques introduced by Dukes and Palutikof ${ }^{35}$ and McNerney and Veers ${ }^{36}$ are used to create the scenarios. In the literature, the Weibull distribution is commonly used to generate synthetic wind speed data. ${ }^{37}$ In that technique, different states are constructed and wind speed is generated using a Markov transition matrix, which is constructed using the Weibull distribution. Wind speed values are centered around the given mean value, and the correlation between time units is handled by a decreasing exponential function.

For each of our low, medium, and high solar and wind cases, 3 scenarios are generated, and their statistics are given in Table 10.

The GCDES, SO, and OMOPSO algorithms are run for 9 different locations (combinations of high, medium, and low resource availability levels) using 9 different scenarios (combinations of 3 solar and 3 wind scenarios), of the data of which are introduced in Section 5.1. The outputs of these computational experiments can be found in Table 11 .

As it is seen among the results, GCDES approach fails to conclude the experiment in a predesignated amount of time for all 9 cases and, hence, delivers only a few solutions. On the other hand, SO method provides a satisfactory number of outcomes after the process is completed. The results indicate that OMOPSO algorithm is able to achieve a variety of solutions with less computational 


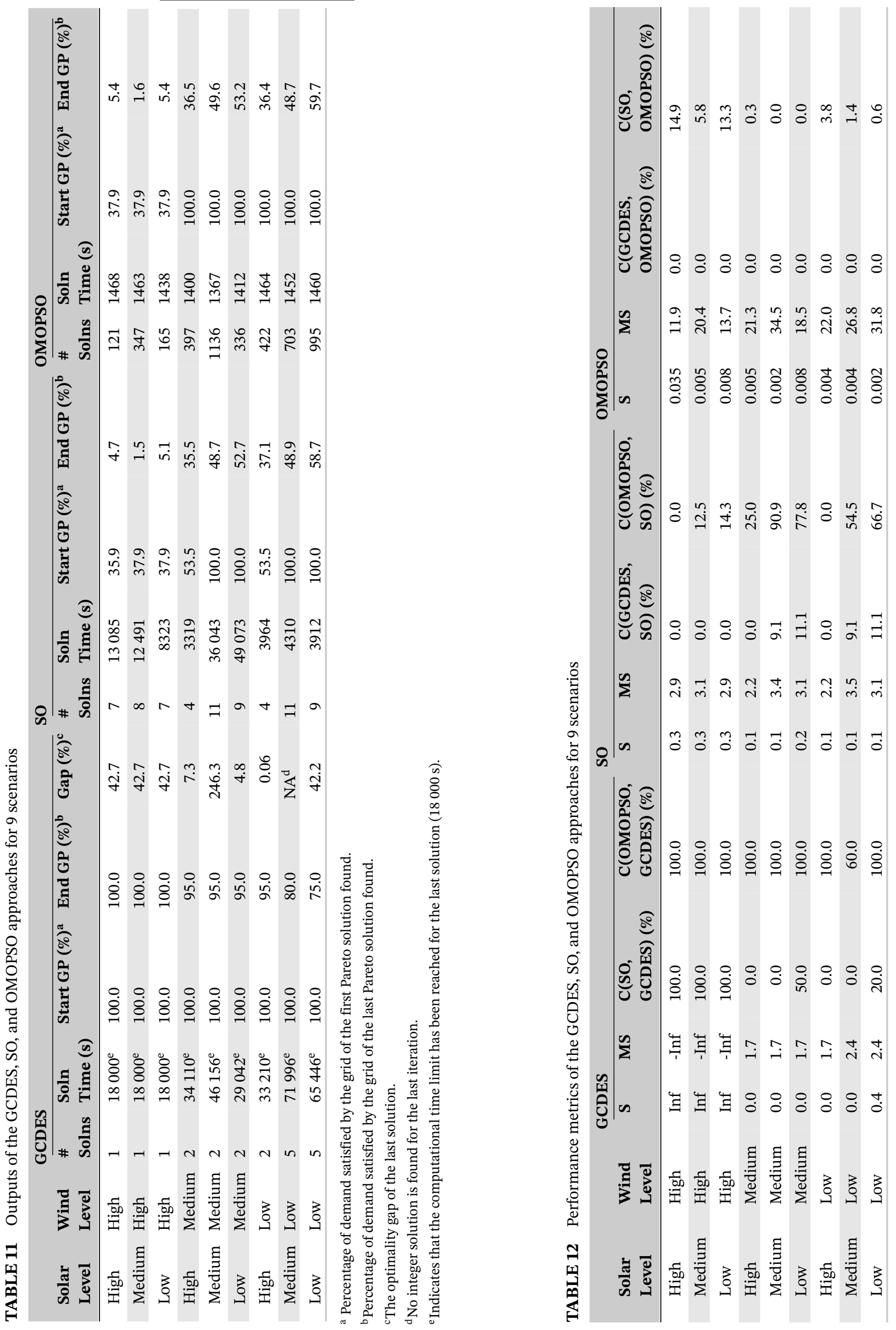


effort compared with the other approaches. Although this table gives valuable insights about the performances, it is necessary to conclude the comparison by looking at the performance measures.

With the 3 metrics implemented, performances of the all approaches can be seen in Table 12. It should be noted that infinity values are recorded because GCDES method finds only one solution in that cases because of time limit. Although this algorithm has $\mathrm{S}$ values as 0 in some settings, the overall performance of the method can be noted as poor when MS and $\mathrm{C}$ numbers are taken into account. While comparing SO and OMOPSO, it is clear that there are significant differences between the numerical results. For the $\mathrm{S}$ measure, OMOPSO has numbers that are almost one-tenth of the SO. A similar ratio is observed among the

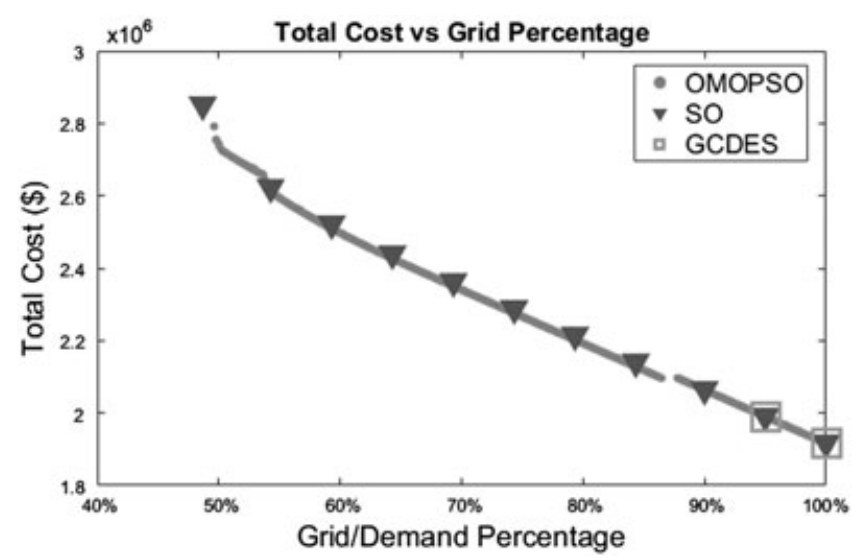

FIGURE 11 Nine-scenario Pareto of the grid-connected decentralized energy system (GCDES), simulation-optimization (SO), and optimized multi-objective particle swarm optimization (OMOPSO) approaches for the medium solar-medium wind case
MS values. Despite high weakly domination ratios in some cases, OMOPSO performs better in terms of the $\mathrm{C}$ metric as well. The results can be seen graphically in Figure 11.

Because of the mediocre performance of the GCDES in 9 scenarios, we now reduce our solution approaches to SO and OMOPSO while working with the scenarios in which demand uncertainty is included. Using medium solar-medium wind case and by generating 3 and 5 scenarios for demand, the number of scenarios is increased to 27 and 125 , respectively. The outcomes of this study can be seen in Table 13. By looking at this table, we can conclude that OMOPSO returns a diverse set of solutions which performs better in all of the metrics in a shorter amount of time.

\section{4 | Sensitivity analysis}

A sensitivity analysis is also conducted to determine the effect of the key parameters on the investment size of the system components for the medium solar-medium wind resource level. For this analysis, we first bound the $\mathrm{CO}_{2}$ level such that the total amount of energy purchased from the grid will not exceed $60 \%$ of the total demand, and solve the problem with our SO and OMOPSO approaches. We investigate the effects of the investment costs of solar, wind, and storage as well as the selling prices of solar and wind energy. For each cost parameter, we halve and double the value (while keeping all other parameters at their base levels). For selling prices, we double the values to see the effect of an increase in the feed-in tariff policy. The results are summarized in Tables 14 and 15. Note that in this problem instance, storing energy is not cost efficient even when the investment cost of storage is halved.

TABLE 13 Outputs of the SO and OMOPSO approaches for 27 to 125 scenarios

\begin{tabular}{|c|c|c|c|c|c|c|c|c|c|c|}
\hline \multirow[b]{2}{*}{ \#Scens } & \multicolumn{5}{|c|}{ SO } & \multicolumn{5}{|l|}{ OMOPSO } \\
\hline & \#Solns & $\begin{array}{l}\text { Soln } \\
\text { Time (s) }\end{array}$ & $\mathbf{S}$ & MS & C(OMOPSO, SO) (\%) & \#Solns & $\begin{array}{l}\text { Soln } \\
\text { Time (s) }\end{array}$ & $\mathbf{S}$ & MS & C(SO, OMOPSO) (\%) \\
\hline 125 & 11 & 976980 & 0.100 & 3.4 & 81.8 & 1056 & 24781 & 0.004 & 33.0 & 1.0 \\
\hline
\end{tabular}

TABLE 14 Sensitivity analysis results of SO approach

\begin{tabular}{|c|c|c|c|c|c|c|c|c|c|}
\hline & \multirow{2}{*}{$\begin{array}{l}\text { Base case } \\
100 \%\end{array}$} & \multicolumn{2}{|l|}{$c_{b}$} & \multicolumn{2}{|l|}{$c_{s}$} & \multicolumn{2}{|l|}{$c_{w}$} & \multirow{2}{*}{$\begin{array}{l}p^{w} \\
200 \%\end{array}$} & \multirow{2}{*}{$\begin{array}{l}p^{s} \\
200 \%\end{array}$} \\
\hline & & $200 \%$ & $50 \%$ & $200 \%$ & $50 \%$ & $200 \%$ & $50 \%$ & & \\
\hline Cost & 2510187 & 2510187 & 2510187 & 2997726 & 1684329 & 2592661 & 1946417 & 2510373 & 2553399 \\
\hline$A_{b}$ & 0 & 0 & 0 & 0 & 0 & 0 & 0 & 0 & 0 \\
\hline$A_{s}$ & 50104 & 50104 & 50104 & 20010 & 97608 & 89713 & 20010 & 31989 & 76666 \\
\hline$A_{w}^{900} \quad \mathrm{~kW}$ & 0 & 0 & 0 & 0 & 0 & 0 & 0 & 0 & 0 \\
\hline$A_{w}^{3} \quad \mathrm{MW}$ & 1 & 1 & 1 & 3 & 0 & 0 & 3 & 2 & 1 \\
\hline
\end{tabular}


TABLE 15 Sensitivity analysis results of OMOPSO approach

\begin{tabular}{|c|c|c|c|c|c|c|c|c|c|}
\hline & \multicolumn{3}{|c|}{ Base case $c_{b}$} & \multicolumn{2}{|l|}{$c_{s}$} & \multicolumn{2}{|l|}{$c_{\underline{w}}$} & \multirow{2}{*}{$\begin{array}{l}p^{w} \\
200 \%\end{array}$} & \multirow{2}{*}{$\begin{array}{l}p^{s} \\
200 \%\end{array}$} \\
\hline & $100 \%$ & $200 \%$ & $50 \%$ & $200 \%$ & $50 \%$ & $200 \%$ & $50 \%$ & & \\
\hline Cost & 2497306 & 2496815 & 2497882 & 3233194 & 1969693 & 2908045 & 2156122 & 2495758 & 2478357 \\
\hline$A_{b}$ & 3.87 & 0 & 0 & 0 & 0 & 0 & 8.96 & 0 & 3.35 \\
\hline$A_{s}$ & 48368 & 48336 & 48482 & 34620 & 65212 & 58636 & 31547 & 48389 & 48336 \\
\hline$A_{w}^{900} \mathrm{~kW}$ & 0 & 0 & 0 & 0 & 0 & 0 & 1 & 0 & 0 \\
\hline$A_{w}^{3} \quad \mathrm{MW}$ & 1 & 1 & 1 & 1 & 0 & 0 & 1 & 1 & 1 \\
\hline
\end{tabular}

\section{CONCLUSION}

Motivated by the interest in shifting from fossil fuel-based centralized energy systems to decentralized renewable energy systems to decrease emissions, we consider the sizing problem of a GC decentralized system. This system is a hybrid decentralized system that depends on solar and wind energy generation backed up with a storage. Our main aim is to provide insights for decision makers about the optimal scale of the decentralized system they plan to invest in. The optimal sizing decision problem includes 2 important aspects, stochasticity (uncertainty of renewable resources) and a multi-objective structure (having concerns for multiple criteria such as cost and emissions). In our study, we consider these 2 aspects elaborately by assuming that the decision maker is responsive to cost and environment (carbon emission) alike and by modeling the problem as a stochastic problem, using random resource availabilities.

We provide a mathematical programming formulation. We then discuss alternative solution approaches that include a simulation-optimization approach and metaheuristic algorithms. We first model the problem as a bi-objective 2-stage stochastic mixed-integer program. This model has 2 objective functions: minimizing the annualized system cost as well as the amount of emitted $\mathrm{CO}_{2}$-equivalent gases while satisfying local demand. Nondominated solutions of this model are generated using the $\varepsilon$-constraint method. This model determines optimal component sizes for a predetermined $\mathrm{CO}_{2}$ emission limit and also determines optimal operational decisions, such as selling, outsourcing, and storing energy in each time unit. Further, we develop a simulation-optimization method, which approaches the multi-stage stochastic nature of the problem in a more realistic way. Preserving the simulation module of this newly introduced method, 3 well-known metaheuristic algorithms are investigated additionally.

A numerical analysis of all methods is performed for multiple-scenario cases. The outputs of the study indicate that the GCDES approach cannot find the whole Pareto set in a reasonable time. Simulation-optimization is better at returning various solutions, however, is outperformed by OMOPSO, which returns better quality solutions in less time.

As future research, this study can be extended in multiple directions. One extension would be to consider more objectives, such as reliability and social acceptance. Interaction among these objectives can provide valuable insights for the decision maker. Another research direction worth exploring is considering ways to increase the uncertainty in the problem. We envisage a potential extension in this direction: One can assume that price parameters are also uncertain. As the number of uncertain parameters increases, the models will become more realistic, yet harder to solve. This situation provides the opportunity to investigate methodologies to tackle the computational challenges as well as to demonstrate the value of stochasticity in such cases. Moreover, one can also consider alternative multi-objective metaheuristic approaches such as multi-objective gravitational search and multi-objective harmony search in the optimization module of our algorithm.

\section{ORCID}

\section{Özlem Karsu (D http://orcid.org/0000-0002-9926-2021}

\section{REFERENCES}

1. World Alliance for Decentralized Energy. Policy to promote de. http://www.localpower.org/pol\&uscore;policies.html, Accessed July 2016.

2. Tina G, Gagliano S. Probabilistic analysis of weather data for a hybrid solar/wind energy system. Int J Energy Res. 2011;35(3):221-232.

3. Kaundinya DP, Balachandra P, Ravindranath NH. Grid-connected versus stand-alone energy systems for decentralized power-a review of literature. Renew Sustainable Energy Rev. 2009;13(8):2041-2050.

4. Jebaraj S, Iniyan S. A review of energy models. Renew sustainable energy rev. 2006;10(4):281-311.

5. Hiremath RB, Shikha S, Ravindranath NH. Decentralized energy planning; modeling and application-a review. Renew Sustainable Energy Rev. 2007;11(5):729-752.

6. Ardakani FJ, Riahy G, Abedi M. Optimal sizing of a grid-connected hybrid system for north-west of iran-case study. Prague, Czech Republic; 2010:29-32. 
7. Kuznia L, Zeng B, Centeno G, Miao Z. Stochastic optimization for power system configuration with renewable energy in remote areas. Ann Oper Res. 2013;210(1):411-432.

8. Gonzalez A, Riba J-R, Rius A, Puig R. Optimal sizing of a hybrid grid-connected photovoltaic and wind power system. Appl Energy. 2015;154:752-762.

9. Bortolini M, Gamberi M, Graziani A. Technical and economic design of photovoltaic and battery energy storage system. Energy Convers Manage. 2014;86:81-92.

10. Chedid R, Rahman S. Unit sizing and control of hybrid wind-solar power systems. IEEE $T$ Energy Conver. 1997;12(1):79-85.

11. Wang L, Singh C. PSO-based multi-criteria optimum design of a grid-connected hybrid power system with multiple renewable sources of energy. In: 2007 IEEE Swarm Intelligence Symposium, Honolulu, HI, USA; 2007:250-257.

12. Perera ATD, Attalage RA, Perera KKCK. Optimal design of a grid connected hybrid electrical energy system using evolutionary computation. In: 2013 IEEE 8th International Conference on Industrial and Information Systems, Peradeniya, Sri Lanka; 2013:12-17.

13. Sharafi M, ElMekkawy TY, Bibeau EL. Optimal design of hybrid renewable energy systems in buildings with low to high renewable energy ratio. Renew Energy. 2015;83:1026-1042.

14. Sharafi M, ElMekkawy TY. Stochastic optimization of hybrid renewable energy systems using sampling average method. Renew Sustainable Energy Rev. 2015;52:1668-1679.

15. Sharafi M, ElMekkawy TY. A dynamic MOPSO algorithm for multiobjective optimal design of hybrid renewable energy systems. Int J Energy Res. 2014;38(15):1949-1963.

16. Powell WB, George A, Simao H, Scott W, Lamont A, Stewart J. Smart: a stochastic multiscale model for the analysis of energy resources, technology, and policy. INFORMS J Comput. 2012;24(4):665-682.

17. Kocaman AS, Abad C, Troy TJ, Huh WT, Modi V. A stochastic model for a macroscale hybrid renewable energy system. Renew Sustainable Energy Rev. 2016;54:688-703.

18. K International. Taxes and incentives for renewable energy. https://assets.kpmg.com/content/dam/kpmg/pdf/2015/09/ taxes-and-incentives-2015-web-v2.pdf, Accessed June 2016; 2015.

19. Birge JR, Louveaux F. Introduction to Stochastic Programming. New York, NY: Springer New York; 2011.

20. Deb K. Multi-Objective Optimization using Evolutionary Algorithms, Vol. 16: In Multi-objective Evolutionary Optimisation for Product Design and Manufacturing. Wang L., Ng AHC and Deb K. eds. London: Springer London; 2001:3-34.

21. Eberhart R, Kennedy J. A new optimizer using particle swarm theory. In: Proceedings of the Sixth International Symposium on Micro Machine and Human Science, 1995. MHS'95, Nagoya, Japan, Japan; 1995:39-43.

22. Coello CAC, Lechuga MS. MOPSO: A proposal for multiple objective particle swarm optimization. In: Proceedings of the 2002 Congress on Evolutionary Computation, 2002. CEC '02, Vol. 2. Honolulu, HI, USA, 2002:1051-1056.
23. Durillo AJ, García-Nieto J, Nebro AJ, Coello CAC, Luna F, Alba E. Multi-objective particle swarm optimizers: An experimental comparison. In: Evolutionary Multi-Criterion Optimization, Vol. 5467, Ehrgott, M. Fonseca, CM Gandibleux X, Hao J-K, and Sevaux M, Eds. Berlin, Heidelberg: Springer Berlin Heidelberg; 2009:495-509.

24. Deb K, Pratap A, Agarwal S, Meyarivan TAMT. A fast and elitist multiobjective genetic algorithm: NSGA-II. IEEE T Evolut Comput. 2002;6(2):182-197.

25. Zitzler E, Laumanns M, Thiele L. SPEA2: Improving the strength pareto evolutionary algorithm; 2001.

26. ENERCON. Enercon product overview. http://www.enercon. de/fileadmin/Redakteur/Medien-Portal/broschueren/pdf/en/ ENERCON_Produkt_en_06_2015.pdf, Accessed June 2016; 2015.

27. Altıntas O. Bi-objective optimization of grid-connected decentralized energy systems. Ms Thesis: Bilkent University; 2016.

28. Source code. https://github.com/MOEAFramework/MOEA Framework/releases/download/v2.12/MOEAFramework-2. 12-Source.tar.gz, Accessed May 2017.

29. Deb K. Multi-objective optimisation using evolutionary algorithms: An introduction; 2011.

30. Schott JR. Fault tolerant design using single and multicriteria genetic algorithm optimization [techincal report]. DTIC Document; 1995.

31. Zitzler E. Evolutionary algorithms for multiobjective optimization: Methods and applications [PhD thesis]. Switzerland: ETH Zurich; 1999.

32. Zitzler E, Thiele L. Multiobjective evolutionary algorithms: a comparative case study and the strength Pareto approach. IEEE T Evolut Comput. 1999;3(4):257-271.

33. Hadka D. Beginner's Guide to the MOEA Framework. 2.8 edition. CreateSpace Independent Publishing Platform, 2016.

34. Examples. htmlexample2, Accessed May 2017.

35. Dukes MDG, Palutikof JP. Estimation of extreme wind speeds with very long return periods. J Appl Meteorol. 1995;34(9):1950-1961.

36. McNerney GM, Veers PS. A Markov Method for Simulating Non-Gaussian Wind Speed Time Series: Wind America, Albuquerque, NM: Sandia National Labs., Albuquerque, NM (USA); 1985.

37. Conradsen K, Nielsen LB, Prahm LP. Review of Weibull statistics for estimation of wind speed distributions. J Clim Appl Meteorol. 1984;23(8):1173-1183.

How to cite this article: Altintas $\mathrm{O}$, Okten $\mathrm{B}$, Karsu O, Kocaman AS. Bi-objective optimization of a grid-connected decentralized energy system. Int $J$ Energy Res. 2018;42:447-465. https://doi.org/10.1002/er.3813 